\title{
High-throughput Designing and Investigation of D-A- $\pi$-A Type Donor Materials for Potential Application in Greenhouse-integrated Solar Cells
}

Muhammad Haroon ${ }^{1}$ and Muhammad Ramzan Saeed Ashraf Janjua ${ }^{1 *}$

'Department of Chemistry, King Fahd University of Petroleum and Minerals, Dhahran 31261, Kingdom of Saudi Arabia

${ }^{*}$ Corresponding author E-mail address:

(Prof. M.R.S.A. Janjua) Janjua@kfupm.edu.sa; Dr_Janjua2010@yahoo.com

Contact \#: +966550442019\& +923006604948

$* * * * * * * * * * * * * * * * * * * * * * * * * * * * * * * * * * * * * * * * * * * * * * * * * * * * * * * * * * * * * * * * *$

\section{Cartesian coordinate of all molecules (R, GH1-GH5)}

R
Symbol
$\mathbf{X}$
Y
Z
C
10.01440000
0.96320000
0.44360000
C
8.69080000
1.05380000
0.44260000
C
8.2582000
$-0.13260000$
0.01050000
$\mathrm{N}$
9.21450000
$-0.95730000$
$-0.25180000$
C
10.27610000
$-0.27710000$
0.02070000
S
7.66940000
2.03540000
0.76050000
C
6.48940000
1.21660000
0.41150000
C
$\begin{array}{llll}6.92320000 & 0.00510000 & -0.01300000\end{array}$
$\mathrm{C}$
$11.61480000 \quad-0.35950000$
0.07190000
C
12.19660000
0.78730000
0.49000000
$\mathrm{S}$
11.15710000
1.80130000
0.76830000
C
5.1962000
1.63210000
0.50390000
C
13.52420000
1.01050000
0.65520000
C
4.18880000
0.79750000
0.17780000
$\mathrm{C}$
2.88950000
1.12240000
0.25460000
C
2.43860000
2.32050000
0.67680000 


\begin{tabular}{|c|c|c|c|}
\hline $\mathrm{C}$ & 3.43940000 & 3.17780000 & 0.98460000 \\
\hline $\mathrm{C}$ & 4.75670000 & 2.85130000 & 0.90330000 \\
\hline $\mathrm{N}$ & 3.24960000 & 4.37970000 & 1.34090000 \\
\hline S & 4.68400000 & 5.22860000 & 1.66390000 \\
\hline $\mathrm{N}$ & 5.51670000 & 3.81200000 & 1.23620000 \\
\hline $\mathrm{C}$ & 1.10230000 & 2.56680000 & 0.77060000 \\
\hline $\mathrm{C}$ & 0.54940000 & 3.52680000 & 1.53850000 \\
\hline $\mathrm{C}$ & -0.77360000 & 3.74460000 & 1.62700000 \\
\hline $\mathrm{C}$ & -1.70860000 & 3.03740000 & 0.95630000 \\
\hline $\mathrm{C}$ & -1.14890000 & 2.07440000 & 0.19210000 \\
\hline $\mathrm{C}$ & 0.17260000 & 1.84510000 & 0.11070000 \\
\hline $\mathbf{N}$ & -2.98530000 & 3.25080000 & 1.03960000 \\
\hline$C$ & -3.76170000 & 2.93500000 & 0.04900000 \\
\hline $\mathrm{C}$ & -3.48510000 & 3.78050000 & 2.11360000 \\
\hline $\mathrm{C}$ & -4.62490000 & 4.51340000 & 2.12110000 \\
\hline$C$ & -5.15220000 & 5.07000000 & 3.22350000 \\
\hline $\mathrm{C}$ & -4.55360000 & 4.91440000 & 4.41010000 \\
\hline $\mathrm{C}$ & -3.42590000 & 4.19590000 & 4.45850000 \\
\hline 4 & -2.91960000 & 3.65480000 & 3.33870000 \\
\hline 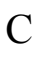 & 9.13170000 & -2.17020000 & -0.68040000 \\
\hline$C$ & 10.18400000 & -2.91190000 & -1.08290000 \\
\hline $\mathrm{C}$ & 10.10940000 & -4.17740000 & -1.52840000 \\
\hline$C$ & 8.95170000 & -4.85600000 & -1.62960000 \\
\hline 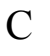 & 7.88800000 & -4.13170000 & -1.23600000 \\
\hline $\mathrm{C}$ & 7.98270000 & -2.86840000 & -0.78800000 \\
\hline $\mathrm{C}$ & 8.86610000 & -6.13640000 & -2.08140000 \\
\hline $\mathrm{C}$ & 7.80200000 & -6.50860000 & -2.84760000 \\
\hline $\mathrm{C}$ & 7.76280000 & -7.77740000 & -3.29960000 \\
\hline
\end{tabular}




\begin{tabular}{|c|c|c|c|}
\hline $\mathrm{C}$ & 8.69650000 & -8.67590000 & -2.97700000 \\
\hline $\mathrm{C}$ & 9.71570000 & -8.29460000 & -2.20320000 \\
\hline$C$ & 9.84590000 & -7.02830000 & -1.76110000 \\
\hline $\mathrm{C}$ & 14.45610000 & 0.07130000 & 0.39630000 \\
\hline $\mathrm{C}$ & 15.77180000 & 0.28490000 & 0.56150000 \\
\hline $\mathrm{C}$ & 16.27380000 & 1.45730000 & 1.00020000 \\
\hline $\mathrm{C}$ & 15.34870000 & 2.39840000 & 1.25660000 \\
\hline $\mathrm{C}$ & 14.03260000 & 2.18330000 & 1.09060000 \\
\hline $\mathrm{C}$ & 17.61410000 & 1.58020000 & 1.13940000 \\
\hline $\mathrm{C}$ & 18.39590000 & 2.60300000 & 1.54850000 \\
\hline $\mathrm{C}$ & -5.06980000 & 2.61530000 & 0.20150000 \\
\hline $\mathrm{C}$ & -5.88470000 & 2.28350000 & -0.81280000 \\
\hline $\mathrm{C}$ & -5.42290000 & 2.25780000 & -2.06840000 \\
\hline $\mathrm{C}$ & -4.13770000 & 2.56800000 & -2.27440000 \\
\hline $\mathrm{C}$ & -3.34520000 & 2.89160000 & -1.23980000 \\
\hline $\mathrm{O}$ & 10.87170000 & -6.67880000 & -0.90820000 \\
\hline $\mathrm{C}$ & 11.54370000 & -7.67940000 & -0.17750000 \\
\hline $\mathrm{O}$ & 6.86120000 & -5.58440000 & -3.25120000 \\
\hline $\mathrm{C}$ & 6.10530000 & -5.82100000 & -4.41710000 \\
\hline $\mathrm{C}$ & 19.75840000 & 2.44930000 & 1.59570000 \\
\hline $\mathrm{O}$ & 20.43600000 & 3.38940000 & 1.96940000 \\
\hline $\mathrm{O}$ & 20.39780000 & 1.30170000 & 1.24780000 \\
\hline $\mathrm{C}$ & 17.91410000 & 3.77760000 & 1.91780000 \\
\hline $\mathrm{N}$ & 17.48590000 & 4.80600000 & 2.24110000 \\
\hline $\mathrm{H}$ & 6.24610000 & -0.77390000 & -0.36100000 \\
\hline $\mathrm{H}$ & 12.19420000 & -1.25280000 & -0.16280000 \\
\hline $\mathrm{H}$ & 4.36690000 & -0.23550000 & -0.15690000 \\
\hline $\mathrm{H}$ & 2.20670000 & 0.29730000 & -0.00270000 \\
\hline
\end{tabular}


Supporting Information file

\begin{tabular}{|c|c|c|c|}
\hline $\mathrm{H}$ & 1.15040000 & 4.17980000 & 2.18660000 \\
\hline $\mathrm{H}$ & -1.05270000 & 4.60880000 & 2.25540000 \\
\hline $\mathrm{H}$ & -1.77290000 & 1.35540000 & -0.36750000 \\
\hline $\mathrm{H}$ & 0.44450000 & 1.02150000 & -0.56830000 \\
\hline $\mathrm{H}$ & -5.15970000 & 4.76800000 & 1.18980000 \\
\hline $\mathrm{H}$ & -6.07100000 & 5.67740000 & 3.15540000 \\
\hline Н & -4.97780000 & 5.36510000 & 5.32220000 \\
\hline $\mathrm{H}$ & -2.92750000 & 4.03380000 & 5.42960000 \\
\hline $\mathrm{H}$ & -2.03120000 & 3.02220000 & 3.50800000 \\
\hline $\mathrm{H}$ & 11.19650000 & -2.50450000 & -1.13220000 \\
\hline $\mathrm{H}$ & 11.05390000 & -4.61500000 & -1.89260000 \\
\hline $\mathrm{H}$ & 6.88360000 & -4.58460000 & -1.18720000 \\
\hline $\mathrm{H}$ & 7.02410000 & -2.46460000 & -0.45400000 \\
\hline $\mathrm{H}$ & 6.95190000 & -8.13470000 & -3.95300000 \\
\hline $\mathrm{H}$ & 8.62710000 & -9.71420000 & -3.34320000 \\
\hline $\mathrm{H}$ & 10.45070000 & -9.07450000 & -1.95090000 \\
\hline $\mathrm{H}$ & 14.18880000 & -0.93250000 & 0.03190000 \\
\hline $\mathrm{H}$ & 16.44150000 & -0.56000000 & 0.31830000 \\
\hline $\mathrm{H}$ & 15.62330000 & 3.39700000 & 1.61820000 \\
\hline $\mathrm{H}$ & 13.38300000 & 3.04010000 & 1.33910000 \\
\hline $\mathrm{H}$ & 18.15760000 & 0.66130000 & 0.86310000 \\
\hline $\mathrm{H}$ & -5.53660000 & 2.53130000 & 1.19810000 \\
\hline $\mathrm{H}$ & -6.93530000 & 2.00890000 & -0.61590000 \\
\hline $\mathrm{H}$ & -6.08270000 & 1.98860000 & -2.90930000 \\
\hline $\mathrm{H}$ & -3.74030000 & 2.57620000 & -3.30380000 \\
\hline 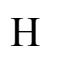 & -2.32720000 & 3.20060000 & -1.53380000 \\
\hline $\mathrm{H}$ & 12.19870000 & -8.28170000 & -0.84510000 \\
\hline & 12.19950000 & -7.17190000 & 0.56540000 \\
\hline
\end{tabular}




$\begin{array}{llll}\mathrm{H} & 10.81390000 & -8.31160000 & 0.37600000 \\ \mathrm{H} & 5.54490000 & -4.88790000 & -4.65110000 \\ \mathrm{H} & 6.77660000 & -6.04900000 & -5.27500000 \\ \mathrm{H} & 5.35700000 & -6.62590000 & -4.24470000 \\ \mathrm{H} & 19.78450000 & 0.60940000 & 0.96400000\end{array}$

\section{GH1}

$\begin{array}{lccl}\text { Symbol } & \mathbf{X} & \mathbf{Y} & \mathbf{Z} \\ \mathrm{C} & 9.14310000 & 0.82340000 & -0.19740000 \\ \mathrm{C} & 7.82550000 & 0.81350000 & -0.04280000 \\ \mathrm{C} & 7.44810000 & -0.44100000 & -0.29640000 \\ \mathrm{~N} & 8.43520000 & -1.21720000 & -0.59160000 \\ \mathrm{C} & 9.45840000 & -0.43420000 & -0.52970000 \\ \mathrm{~S} & 6.77310000 & 1.74980000 & 0.30730000 \\ \mathrm{C} & 5.63340000 & 0.81560000 & 0.19340000 \\ \mathrm{C} & 6.11350000 & -0.40190000 & -0.15870000 \\ \mathrm{C} & 10.79460000 & -0.40940000 & -0.65280000 \\ \mathrm{C} & 11.28520000 & 0.83240000 & -0.46040000 \\ \mathrm{~S} & 10.22720000 & 1.78780000 & -0.08400000 \\ \mathrm{C} & 4.33020000 & 1.14690000 & 0.40680000 \\ \mathrm{C} & 12.60130000 & 1.12410000 & -0.46430000 \\ \mathrm{C} & 3.36300000 & 0.21540000 & 0.28490000 \\ \mathrm{C} & 2.05810000 & 0.45750000 & 0.48020000 \\ \mathrm{C} & 1.56040000 & 1.66600000 & 0.80910000 \\ \mathrm{C} & 2.52040000 & 2.60950000 & 0.94700000 \\ \mathrm{C} & 3.84390000 & 2.36380000 & 0.75640000 \\ \mathrm{~N} & 2.28600000 & 3.80140000 & 1.31040000 \\ & 3.67760000 & 4.76940000 & 1.40580000\end{array}$




\begin{tabular}{|c|c|c|c|}
\hline $\mathrm{N}$ & 4.56120000 & 3.39340000 & 0.94840000 \\
\hline $\mathrm{C}$ & 0.21950000 & 1.84730000 & 0.96360000 \\
\hline $\mathrm{C}$ & -0.63440000 & 0.83170000 & 1.20780000 \\
\hline $\mathrm{C}$ & -1.95770000 & 0.99280000 & 1.37630000 \\
\hline $\mathrm{C}$ & -2.59510000 & 2.18120000 & 1.29940000 \\
\hline $\mathrm{C}$ & -1.73770000 & 3.18960000 & 1.03040000 \\
\hline $\mathrm{C}$ & -0.41170000 & 3.03490000 & 0.87630000 \\
\hline $\mathrm{N}$ & -3.87360000 & 2.33150000 & 1.45800000 \\
\hline $\mathrm{C}$ & -4.48690000 & 3.31900000 & 0.88160000 \\
\hline $\mathrm{C}$ & -4.53710000 & 1.49470000 & 2.19520000 \\
\hline $\mathrm{C}$ & -5.85370000 & 1.22730000 & 2.01760000 \\
\hline $\mathrm{C}$ & -6.55220000 & 0.36280000 & 2.77120000 \\
\hline $\mathrm{C}$ & -5.95570000 & -0.29440000 & 3.77250000 \\
\hline $\mathrm{C}$ & -4.65590000 & -0.06480000 & 3.99190000 \\
\hline $\mathrm{C}$ & -3.98180000 & 0.80200000 & 3.21910000 \\
\hline $\mathrm{C}$ & 8.41130000 & -2.47310000 & -0.88110000 \\
\hline $\mathrm{C}$ & 9.48130000 & -3.18000000 & -1.29930000 \\
\hline $\mathrm{C}$ & 9.47100000 & -4.48940000 & -1.60070000 \\
\hline $\mathrm{C}$ & 8.36640000 & -5.25420000 & -1.52370000 \\
\hline $\mathrm{C}$ & 7.28550000 & -4.56700000 & -1.11090000 \\
\hline $\mathrm{C}$ & 7.31720000 & -3.25850000 & -0.80700000 \\
\hline $\mathrm{C}$ & 8.34510000 & -6.57950000 & -1.82990000 \\
\hline $\mathrm{C}$ & 7.25440000 & -7.10640000 & -2.45490000 \\
\hline $\mathrm{C}$ & 7.27830000 & -8.41700000 & -2.76750000 \\
\hline $\mathrm{C}$ & 8.30270000 & -9.20760000 & -2.43800000 \\
\hline $\mathrm{C}$ & 9.34840000 & -8.67370000 & -1.80200000 \\
\hline $\mathrm{C}$ & 9.41450000 & -7.36050000 & -1.50730000 \\
\hline $\mathrm{C}$ & -5.62260000 & 3.87580000 & 1.36810000 \\
\hline
\end{tabular}




\begin{tabular}{|c|c|c|c|}
\hline $\mathrm{C}$ & -6.26680000 & 4.89890000 & 0.78390000 \\
\hline $\mathrm{C}$ & -5.79900000 & 5.43040000 & -0.35150000 \\
\hline $\mathrm{C}$ & -4.68090000 & 4.91520000 & -0.87560000 \\
\hline $\mathrm{C}$ & -4.05550000 & 3.89490000 & -0.26670000 \\
\hline $\mathrm{C}$ & 14.48070000 & 2.32210000 & 0.18640000 \\
\hline $\mathrm{C}$ & 14.80370000 & 3.62840000 & 0.31130000 \\
\hline $\mathrm{C}$ & 13.83040000 & 4.39360000 & -0.19640000 \\
\hline $\mathrm{C}$ & 12.88600000 & 3.57860000 & -0.71640000 \\
\hline $\mathrm{C}$ & 15.92060000 & 4.12290000 & 0.86180000 \\
\hline $\mathrm{C}$ & 16.07070000 & 5.45660000 & 0.90800000 \\
\hline $\mathrm{C}$ & 15.10800000 & 6.25340000 & 0.40280000 \\
\hline $\mathrm{C}$ & 14.00050000 & 5.72510000 & -0.15000000 \\
\hline $\mathrm{O}$ & 15.15560000 & 1.38860000 & 0.56450000 \\
\hline $\mathrm{C}$ & 11.88080000 & 3.98190000 & -1.52500000 \\
\hline $\mathrm{F}$ & 15.25000000 & 7.57120000 & 0.44170000 \\
\hline $\mathrm{F}$ & 17.16350000 & 5.97590000 & 1.44870000 \\
\hline $\mathrm{C}$ & 13.23510000 & 2.31280000 & -0.36980000 \\
\hline $\mathrm{O}$ & 10.47550000 & -6.84840000 & -0.79020000 \\
\hline $\mathrm{C}$ & 11.28250000 & -7.71350000 & -0.02390000 \\
\hline $\mathrm{C}$ & 11.60660000 & 5.23540000 & -1.83220000 \\
\hline $\mathrm{N}$ & 11.35420000 & 6.32760000 & -2.12940000 \\
\hline $\mathrm{C}$ & 11.22140000 & 3.14210000 & -2.30550000 \\
\hline $\mathrm{N}$ & 10.71150000 & 2.42020000 & -3.05800000 \\
\hline $\mathrm{O}$ & 6.21460000 & -6.29940000 & -2.86660000 \\
\hline $\mathrm{C}$ & 5.39070000 & -6.71290000 & -3.93280000 \\
\hline $\mathrm{H}$ & 5.46730000 & -1.26150000 & -0.32930000 \\
\hline $\mathrm{H}$ & 11.46700000 & -1.24250000 & -0.87920000 \\
\hline $\mathrm{H}$ & 13.24000000 & 0.22100000 & -0.45520000 \\
\hline
\end{tabular}




\begin{tabular}{|c|c|c|c|}
\hline $\mathrm{H}$ & 3.58110000 & -0.82490000 & 0.00030000 \\
\hline$H$ & 1.40780000 & -0.41220000 & 0.29460000 \\
\hline $\mathrm{H}$ & -0.29040000 & -0.20920000 & 1.31580000 \\
\hline $\mathrm{H}$ & -2.51900000 & 0.05210000 & 1.51690000 \\
\hline $\mathrm{H}$ & -2.08320000 & 4.23760000 & 0.98790000 \\
\hline $\mathrm{H}$ & 0.11950000 & 3.96530000 & 0.63070000 \\
\hline 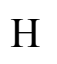 & -6.42670000 & 1.65500000 & 1.17690000 \\
\hline $\mathrm{H}$ & -7.61790000 & 0.17230000 & 2.55680000 \\
\hline $\mathrm{H}$ & -6.51910000 & -1.00490000 & 4.39910000 \\
\hline $\mathrm{H}$ & -4.14810000 & -0.57760000 & 4.82680000 \\
\hline $\mathrm{H}$ & -2.93800000 & 0.96850000 & 3.53690000 \\
\hline $\mathrm{H}$ & 10.45300000 & -2.71310000 & -1.47420000 \\
\hline $\mathrm{H}$ & 10.41440000 & -4.89860000 & -1.99890000 \\
\hline $\mathrm{H}$ & 6.32730000 & -5.08070000 & -0.92540000 \\
\hline $\mathrm{H}$ & 6.35900000 & -2.88790000 & -0.43700000 \\
\hline $\mathrm{H}$ & 6.44730000 & -8.89910000 & -3.30520000 \\
\hline $\mathrm{H}$ & 8.28530000 & -10.28230000 & -2.68670000 \\
\hline 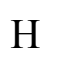 & 10.16030000 & -9.36850000 & -1.53660000 \\
\hline $\mathrm{H}$ & -6.05470000 & 3.56670000 & 2.33550000 \\
\hline $\mathrm{H}$ & -7.17450000 & 5.32230000 & 1.24700000 \\
\hline $\mathrm{H}$ & -6.32030000 & 6.26890000 & -0.84130000 \\
\hline $\mathrm{H}$ & -4.28950000 & 5.32170000 & -1.82400000 \\
\hline $\mathrm{H}$ & -3.18840000 & 3.50210000 & -0.82530000 \\
\hline $\mathrm{H}$ & 16.70260000 & 3.46530000 & 1.27340000 \\
\hline $\mathrm{H}$ & 13.25750000 & 6.42750000 & -0.54790000 \\
\hline $\mathrm{H}$ & 11.92550000 & -8.33860000 & -0.68220000 \\
\hline $\mathrm{H}$ & 11.95550000 & -7.08300000 & 0.59990000 \\
\hline & 10.65320000 & -8.33020000 & 0.65600000 \\
\hline
\end{tabular}




\begin{tabular}{|c|c|c|c|}
\hline $\mathrm{H}$ & 4.74270000 & -5.85180000 & -4.21230000 \\
\hline $\mathrm{H}$ & 6.00870000 & -6.98590000 & -4.81730000 \\
\hline & 4.72310000 & -7.54420000 & -3.61520000 \\
\hline
\end{tabular}

\section{GH2}

$\begin{array}{lccc}\text { Symbol } & \text { X } & \text { Y Z } \\ \text { C } & 9.13930000 & 0.82010000 & -0.18780000 \\ \text { C } & 7.82180000 & 0.80890000 & -0.03320000 \\ \text { C } & 7.44520000 & -0.44520000 & -0.28950000 \\ \text { N } & 8.43280000 & -1.22010000 & -0.58590000 \\ \text { C } & 9.45550000 & -0.43670000 & -0.52240000 \\ \text { S } & 6.76860000 & 1.74380000 & 0.31880000 \\ \text { C } & 5.62950000 & 0.80930000 & 0.20120000 \\ \text { C } & 6.11040000 & -0.40730000 & -0.15270000 \\ \text { C } & 10.79170000 & -0.41090000 & -0.64520000 \\ \text { C } & 11.28140000 & 0.83080000 & -0.45040000 \\ \text { S } & 10.22280000 & 1.78490000 & -0.07250000 \\ \text { C } & 4.32580000 & 1.13990000 & 0.41310000 \\ \text { C } & 12.59730000 & 1.12310000 & -0.45380000 \\ \text { C } & 3.35900000 & 0.20870000 & 0.28630000 \\ \text { C } & 2.05360000 & 0.45020000 & 0.47880000 \\ \text { C } & 1.55490000 & 1.65780000 & 0.80980000 \\ \text { C } & 2.51470000 & 2.60070000 & 0.95340000 \\ \text { C } & 3.83870000 & 2.35570000 & 0.76530000 \\ \text { N } & 2.27940000 & 3.79150000 & 1.32000000 \\ \text { S } & 3.67070000 & 4.75910000 & 1.42210000 \\ \text { N } & 4.55550000 & 3.38470000 & 0.96240000 \\ \text { N } & 0.21360000 & 1.83880000 & 0.96050000\end{array}$


Supporting Information file

\begin{tabular}{|c|c|c|c|}
\hline $\mathrm{C}$ & -0.64140000 & 0.82250000 & 1.19810000 \\
\hline $\mathrm{C}$ & -1.96530000 & 0.98310000 & 1.36200000 \\
\hline $\mathrm{C}$ & -2.60250000 & 2.17170000 & 1.28700000 \\
\hline $\mathrm{C}$ & -1.74380000 & 3.18090000 & 1.02500000 \\
\hline $\mathrm{C}$ & -0.41730000 & 3.02670000 & 0.87510000 \\
\hline $\mathrm{N}$ & -3.88160000 & 2.32160000 & 1.44080000 \\
\hline $\mathrm{C}$ & -4.49240000 & 3.31150000 & 0.86580000 \\
\hline $\mathrm{C}$ & -4.54840000 & 1.48200000 & 2.17190000 \\
\hline $\mathrm{C}$ & -5.86430000 & 1.21570000 & 1.98780000 \\
\hline $\mathrm{C}$ & -6.56630000 & 0.34840000 & 2.73490000 \\
\hline $\mathrm{C}$ & -5.97400000 & -0.31330000 & 3.73580000 \\
\hline $\mathrm{C}$ & -4.67510000 & -0.08500000 & 3.96150000 \\
\hline $\mathrm{C}$ & -3.99760000 & 0.78490000 & 3.19520000 \\
\hline $\mathrm{C}$ & 8.40970000 & -2.47550000 & -0.87800000 \\
\hline $\mathrm{C}$ & 9.47990000 & -3.18030000 & -1.29920000 \\
\hline $\mathrm{C}$ & 9.47010000 & -4.48900000 & -1.60340000 \\
\hline $\mathrm{C}$ & 8.36640000 & -5.25510000 & -1.52620000 \\
\hline $\mathrm{C}$ & 7.28560000 & -4.56990000 & -1.10990000 \\
\hline $\mathrm{C}$ & 7.31640000 & -3.26190000 & -0.80360000 \\
\hline $\mathrm{C}$ & 8.34550000 & -6.57970000 & -1.83510000 \\
\hline $\mathrm{C}$ & 7.25390000 & -7.10650000 & -2.45850000 \\
\hline $\mathrm{C}$ & 7.27830000 & -8.41640000 & -2.77360000 \\
\hline $\mathrm{C}$ & 8.30430000 & -9.20660000 & -2.44810000 \\
\hline $\mathrm{C}$ & 9.35110000 & -8.67300000 & -1.81360000 \\
\hline $\mathrm{C}$ & 9.41660000 & -7.36030000 & -1.51660000 \\
\hline $\mathrm{C}$ & -5.62990000 & 3.86660000 & 1.34980000 \\
\hline $\mathrm{C}$ & -6.27170000 & 4.89200000 & 0.76710000 \\
\hline$\gamma$ & -5.79910000 & 5.42810000 & -0.36410000 \\
\hline
\end{tabular}




\begin{tabular}{|c|c|c|c|}
\hline $\mathrm{C}$ & -4.67890000 & 4.91490000 & -0.88570000 \\
\hline $\mathrm{C}$ & -4.05620000 & 3.89200000 & -0.27830000 \\
\hline $\mathrm{C}$ & 14.47580000 & 2.32120000 & 0.19940000 \\
\hline $\mathrm{C}$ & 14.79760000 & 3.62760000 & 0.32750000 \\
\hline $\mathrm{C}$ & 13.82390000 & 4.39290000 & -0.17800000 \\
\hline $\mathrm{C}$ & 12.88060000 & 3.57830000 & -0.70100000 \\
\hline $\mathrm{C}$ & 15.91430000 & 4.12090000 & 0.87920000 \\
\hline $\mathrm{C}$ & 16.06430000 & 5.45580000 & 0.92990000 \\
\hline $\mathrm{C}$ & 15.09950000 & 6.25440000 & 0.42710000 \\
\hline $\mathrm{C}$ & 13.99160000 & 5.72460000 & -0.12740000 \\
\hline $\mathrm{O}$ & 15.15090000 & 1.38690000 & 0.57510000 \\
\hline $\mathrm{C}$ & 11.87540000 & 3.98050000 & -1.51030000 \\
\hline $\mathrm{Cl}$ & 15.23560000 & 7.98350000 & 0.46020000 \\
\hline $\mathrm{Cl}$ & 17.51230000 & 6.07940000 & 1.64980000 \\
\hline $\mathrm{C}$ & 13.23040000 & 2.31200000 & -0.35690000 \\
\hline $\mathrm{C}$ & 11.59820000 & 5.23320000 & -1.81800000 \\
\hline $\mathrm{N}$ & 11.34420000 & 6.32500000 & -2.11530000 \\
\hline $\mathrm{C}$ & 11.21930000 & 3.13980000 & -2.29270000 \\
\hline $\mathrm{N}$ & 10.71220000 & 2.41750000 & -3.04660000 \\
\hline $\mathrm{O}$ & 10.47870000 & -6.84850000 & -0.80120000 \\
\hline $\mathrm{C}$ & 11.28870000 & -7.71390000 & -0.03850000 \\
\hline $\mathrm{O}$ & 6.21230000 & -6.29970000 & -2.86620000 \\
\hline $\mathrm{C}$ & 5.38590000 & -6.71190000 & -3.93110000 \\
\hline $\mathrm{H}$ & 5.46490000 & -1.26700000 & -0.32590000 \\
\hline $\mathrm{H}$ & 11.46450000 & -1.24350000 & -0.87300000 \\
\hline $\mathrm{H}$ & 13.23640000 & 0.22010000 & -0.44640000 \\
\hline $\mathrm{H}$ & 3.57790000 & -0.83060000 & -0.00100000 \\
\hline $\mathrm{H}$ & 1.40400000 & -0.41900000 & 0.28890000 \\
\hline
\end{tabular}


Supporting Information file

\begin{tabular}{|c|c|c|c|}
\hline $\mathrm{H}$ & -0.29800000 & -0.21880000 & 1.30360000 \\
\hline $\mathrm{H}$ & -2.52710000 & 0.04190000 & 1.49700000 \\
\hline & -2.08890000 & 4.22920000 & 0.98480000 \\
\hline & 0.11490000 & 3.95780000 & 0.63450000 \\
\hline & -6.43370000 & 1.64710000 & 1.14660000 \\
\hline & -7.63110000 & 0.15930000 & 2.51540000 \\
\hline $\mathrm{H}$ & -6.54020000 & -1.02630000 & 4.35700000 \\
\hline & -4.17100000 & -0.60150000 & 4.79640000 \\
\hline $\mathrm{H}$ & -2.95510000 & 0.94980000 & 3.51820000 \\
\hline $\mathrm{H}$ & 10.45070000 & -2.71190000 & -1.47480000 \\
\hline $\mathrm{H}$ & 10.41330000 & -4.89620000 & -2.00430000 \\
\hline $\mathrm{H}$ & 6.32840000 & -5.08490000 & -0.92340000 \\
\hline $\mathrm{H}$ & 6.35850000 & -2.89320000 & -0.43080000 \\
\hline $\mathrm{H}$ & 6.44650000 & -8.89840000 & -3.31030000 \\
\hline $\mathrm{H}$ & 8.28740000 & -10.28080000 & -2.69890000 \\
\hline $\mathrm{H}$ & 10.16430000 & -9.36740000 & -1.55150000 \\
\hline $\mathrm{H}$ & -6.06610000 & 3.55370000 & 2.31420000 \\
\hline $\mathrm{H}$ & -7.18130000 & 5.31380000 & 1.22820000 \\
\hline H & -6.31820000 & 6.26880000 & -0.85280000 \\
\hline H & -4.28340000 & 5.32530000 & -1.83070000 \\
\hline $\mathrm{H}$ & -3.18700000 & 3.50130000 & -0.83510000 \\
\hline $\mathrm{H}$ & 16.68660000 & 3.44680000 & 1.28380000 \\
\hline $\mathrm{H}$ & 13.23380000 & 6.40950000 & -0.52810000 \\
\hline $\mathrm{H}$ & 11.93140000 & -8.33630000 & -0.69960000 \\
\hline H & 11.96190000 & -7.08360000 & 0.58530000 \\
\hline H & 10.66190000 & -8.33320000 & 0.64130000 \\
\hline $\mathrm{H}$ & 4.73610000 & -5.85090000 & -4.20670000 \\
\hline & 6.00170000 & -6.98210000 & -4.81780000 \\
\hline
\end{tabular}


Supporting Information file

$\mathrm{H} \quad 4.72010000 \quad-7.54460000 \quad-3.61340000$

\section{GH3}

$\begin{array}{lccl}\text { Symbol } & \mathbf{X} & \mathbf{Y} & \mathbf{Z} \\ \mathrm{C} & 9.03200000 & 0.71100000 & -0.12090000 \\ \mathrm{C} & 7.71150000 & 0.68880000 & 0.00420000 \\ \mathrm{C} & 7.34820000 & -0.56110000 & -0.28950000 \\ \mathrm{~N} & 8.34680000 & -1.32310000 & -0.58230000 \\ \mathrm{C} & 9.36320000 & -0.53570000 & -0.47790000 \\ \mathrm{~S} & 6.64530000 & 1.60930000 & 0.35480000 \\ \mathrm{C} & 5.51480000 & 0.67130000 & 0.19120000 \\ \mathrm{C} & 6.01060000 & -0.53380000 & -0.18100000 \\ \mathrm{C} & 10.70160000 & -0.49970000 & -0.57020000 \\ \mathrm{C} & 11.17910000 & 0.73980000 & -0.33550000 \\ \mathrm{~S} & 10.10660000 & 1.67900000 & 0.04110000 \\ \mathrm{C} & 4.20490000 & 0.98910000 & 0.38340000 \\ \mathrm{C} & 12.49270000 & 1.04060000 & -0.30170000 \\ \mathrm{C} & 3.24690000 & 0.05540000 & 0.21530000 \\ \mathrm{C} & 1.93630000 & 0.28450000 & 0.38670000 \\ \mathrm{C} & 1.42320000 & 1.48120000 & 0.73500000 \\ \mathrm{C} & 2.37370000 & 2.42630000 & 0.91970000 \\ \mathrm{C} & 3.70290000 & 2.19350000 & 0.75330000 \\ \mathrm{~N} & 2.12340000 & 3.60680000 & 1.30860000 \\ \mathrm{~S} & 3.50620000 & 4.58010000 & 1.46150000 \\ \mathrm{~N} & 4.40890000 & 3.22190000 & 0.98860000 \\ \mathrm{C} & 0.07800000 & 1.65080000 & 0.86220000 \\ \mathrm{C} & -0.77520000 & 0.62440000 & 1.05920000 \\ \mathrm{C} & -2.10320000 & 0.77380000 & 1.19960000\end{array}$


Supporting Information file

\begin{tabular}{|c|c|c|c|}
\hline $\mathrm{C}$ & -2.74610000 & 1.96010000 & 1.13870000 \\
\hline C & -1.88860000 & 2.97990000 & 0.91690000 \\
\hline$C$ & -0.55840000 & 2.83670000 & 0.79060000 \\
\hline $\mathrm{N}$ & -4.02890000 & 2.09910000 & 1.27020000 \\
\hline C & -4.63450000 & 3.09810000 & 0.70560000 \\
\hline C & -4.70460000 & 1.23910000 & 1.96880000 \\
\hline $\mathrm{C}$ & -6.01490000 & 0.96930000 & 1.75260000 \\
\hline $\mathrm{C}$ & -6.72590000 & 0.08110000 & 2.46570000 \\
\hline C & -6.14930000 & -0.59970000 & 3.46290000 \\
\hline 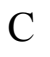 & -4.85650000 & -0.36900000 & 3.71960000 \\
\hline $\mathrm{C}$ & -4.16970000 & 0.52220000 & 2.98690000 \\
\hline$C$ & 8.33790000 & -2.57140000 & -0.90390000 \\
\hline $\mathrm{C}$ & 9.42130000 & -3.25990000 & -1.31810000 \\
\hline $\mathrm{C}$ & 9.42650000 & -4.56120000 & -1.65270000 \\
\hline $\mathrm{C}$ & 8.32640000 & -5.33540000 & -1.61700000 \\
\hline C & 7.23240000 & -4.66640000 & -1.20870000 \\
\hline $\mathrm{C}$ & 7.24830000 & -3.36570000 & -0.87160000 \\
\hline $\mathrm{C}$ & 8.32090000 & -6.65270000 & -1.95670000 \\
\hline $\mathrm{C}$ & 7.24640000 & -7.17180000 & -2.61530000 \\
\hline $\mathrm{C}$ & 7.28630000 & -8.47390000 & -2.95990000 \\
\hline$C$ & 8.31050000 & -9.26500000 & -2.63100000 \\
\hline $\mathrm{C}$ & 9.33980000 & -8.73970000 & -1.96190000 \\
\hline $\mathrm{C}$ & 9.39010000 & -7.43380000 & -1.63330000 \\
\hline 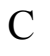 & -5.78500000 & 3.63540000 & 1.17900000 \\
\hline 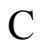 & -6.42150000 & 4.67000000 & 0.60670000 \\
\hline $\mathrm{C}$ & -5.92990000 & 5.23420000 & -0.50260000 \\
\hline $\mathrm{C}$ & -4.79630000 & 4.73950000 & -1.01300000 \\
\hline $\mathrm{C}$ & -4.17930000 & 3.70680000 & -0.41640000 \\
\hline
\end{tabular}




\begin{tabular}{|c|c|c|c|}
\hline $\mathrm{C}$ & 14.34540000 & 2.23650000 & 0.42450000 \\
\hline $\mathrm{C}$ & 14.65320000 & 3.54160000 & 0.59260000 \\
\hline $\mathrm{C}$ & 13.68610000 & 4.31230000 & 0.08270000 \\
\hline $\mathrm{C}$ & 12.76230000 & 3.50330000 & -0.48280000 \\
\hline $\mathrm{C}$ & 15.75230000 & 4.02890000 & 1.18390000 \\
\hline $\mathrm{C}$ & 15.89200000 & 5.36340000 & 1.27330000 \\
\hline $\mathrm{C}$ & 14.93420000 & 6.16690000 & 0.76790000 \\
\hline $\mathrm{C}$ & 13.84380000 & 5.64340000 & 0.17250000 \\
\hline $\mathrm{O}$ & 15.02090000 & 1.30030000 & 0.79440000 \\
\hline $\mathrm{C}$ & 11.77350000 & 3.91720000 & -1.30620000 \\
\hline $\mathrm{C}$ & 13.11370000 & 2.23140000 & -0.16130000 \\
\hline $\mathrm{C}$ & 16.97590000 & 5.83060000 & 1.86080000 \\
\hline $\mathrm{N}$ & 17.93060000 & 6.23620000 & 2.37700000 \\
\hline $\mathrm{C}$ & 15.03280000 & 7.48050000 & 0.83360000 \\
\hline $\mathrm{N}$ & 15.12400000 & 8.63410000 & 0.89350000 \\
\hline $\mathrm{C}$ & 11.14010000 & 3.09280000 & -2.12380000 \\
\hline $\mathrm{N}$ & 10.65460000 & 2.38640000 & -2.90660000 \\
\hline $\mathrm{C}$ & 11.49960000 & 5.17640000 & -1.58830000 \\
\hline $\mathrm{N}$ & 11.25840000 & 6.27800000 & -1.85870000 \\
\hline $\mathrm{O}$ & 10.43330000 & -6.93220000 & -0.88350000 \\
\hline $\mathrm{C}$ & 11.23250000 & -7.81000000 & -0.12380000 \\
\hline $\mathrm{O}$ & 6.20830000 & -6.36230000 & -3.02660000 \\
\hline $\mathrm{C}$ & 5.40760000 & -6.75490000 & -4.11810000 \\
\hline $\mathrm{H}$ & 5.37440000 & -1.39280000 & -0.38830000 \\
\hline $\mathrm{H}$ & 11.38450000 & -1.32280000 & -0.80200000 \\
\hline $\mathrm{H}$ & 13.13830000 & 0.14230000 & -0.30060000 \\
\hline $\mathrm{H}$ & 3.47830000 & -0.97580000 & -0.09120000 \\
\hline $\mathrm{H}$ & 1.29610000 & -0.58390000 & 0.16380000 \\
\hline
\end{tabular}


Supporting Information file

\begin{tabular}{|c|c|c|c|}
\hline $\mathrm{H}$ & -0.42760000 & -0.41710000 & 1.14820000 \\
\hline H & -2.66180000 & -0.17340000 & 1.30170000 \\
\hline $\mathrm{H}$ & -2.23950000 & 4.02670000 & 0.89330000 \\
\hline $\mathrm{H}$ & -0.02720000 & 3.77600000 & 0.58200000 \\
\hline H & -6.57020000 & 1.41620000 & 0.91000000 \\
\hline $\mathrm{H}$ & -7.78490000 & -0.10930000 & 2.22080000 \\
\hline $\mathrm{H}$ & -6.72310000 & -1.33000000 & 4.05640000 \\
\hline $\mathrm{H}$ & -4.36570000 & -0.90160000 & 4.55220000 \\
\hline $\mathrm{H}$ & -3.13480000 & 0.68570000 & 3.33410000 \\
\hline $\mathrm{H}$ & 10.39270000 & -2.78170000 & -1.46180000 \\
\hline $\mathrm{H}$ & 10.38080000 & -4.95320000 & -2.04240000 \\
\hline H & 6.27480000 & -5.19120000 & -1.05490000 \\
\hline $\mathrm{H}$ & 6.28030000 & -3.01120000 & -0.51110000 \\
\hline $\mathrm{H}$ & 6.46940000 & -8.94850000 & -3.52530000 \\
\hline $\mathrm{H}$ & 8.30630000 & -10.33310000 & -2.90680000 \\
\hline $\mathrm{H}$ & 10.15200000 & -9.43480000 & -1.69840000 \\
\hline $\mathrm{H}$ & -6.23820000 & 3.29830000 & 2.12720000 \\
\hline $\mathrm{H}$ & -7.34280000 & 5.07580000 & 1.05870000 \\
\hline $\mathrm{H}$ & -6.44460000 & 6.08250000 & -0.98250000 \\
\hline $\mathrm{H}$ & -4.38480000 & 5.17330000 & -1.94050000 \\
\hline $\mathrm{H}$ & -3.29680000 & 3.33380000 & -0.96420000 \\
\hline $\mathrm{H}$ & 16.52140000 & 3.35210000 & 1.59150000 \\
\hline $\mathrm{H}$ & 13.09320000 & 6.33800000 & -0.22680000 \\
\hline $\mathrm{H}$ & 11.89290000 & -8.41330000 & -0.78510000 \\
\hline $\mathrm{H}$ & 11.88820000 & -7.19030000 & 0.52850000 \\
\hline $\mathrm{H}$ & 10.59540000 & -8.44860000 & 0.52810000 \\
\hline $\mathrm{H}$ & 4.75900000 & -5.89140000 & -4.38860000 \\
\hline $\mathrm{H}$ & 6.04420000 & -7.00170000 & -4.99710000 \\
\hline
\end{tabular}




\section{GH4}

$\begin{array}{lccl}\text { Symbol } & \mathbf{X} & \mathbf{Y} & \mathbf{Z} \\ \mathrm{C} & 8.70090000 & 0.35450000 & 0.09170000 \\ \mathrm{C} & 7.37730000 & 0.30030000 & 0.16160000 \\ \mathrm{C} & 7.05260000 & -0.93850000 & -0.21300000 \\ \mathrm{~N} & 8.07810000 & -1.66400000 & -0.50580000 \\ \mathrm{C} & 9.07260000 & -0.86410000 & -0.31850000 \\ \mathrm{~S} & 6.27930000 & 1.18050000 & 0.51710000 \\ \mathrm{C} & 5.17620000 & 0.23110000 & 0.25930000 \\ \mathrm{C} & 5.71150000 & -0.94260000 & -0.15630000 \\ \mathrm{C} & 10.41250000 & -0.79660000 & -0.35520000 \\ \mathrm{C} & 10.85370000 & 0.43690000 & -0.03380000 \\ \mathrm{~S} & 9.74690000 & 1.33300000 & 0.34910000 \\ \mathrm{C} & 3.85360000 & 0.51350000 & 0.41590000 \\ \mathrm{C} & 12.15840000 & 0.76010000 & 0.07020000 \\ \mathrm{C} & 2.92260000 & -0.42810000 & 0.16160000 \\ \mathrm{C} & 1.60190000 & -0.23310000 & 0.29280000 \\ \mathrm{C} & 1.05090000 & 0.93360000 & 0.68250000 \\ \mathrm{C} & 1.97340000 & 1.88560000 & 0.95310000 \\ \mathrm{C} & 3.31260000 & 1.68710000 & 0.82770000 \\ \mathrm{~N} & 1.68350000 & 3.03910000 & 1.39230000 \\ \mathrm{~S} & 3.03850000 & 4.02920000 & 1.64990000 \\ \mathrm{~N} & 3.98710000 & 2.71500000 & 1.14360000 \\ \mathrm{C} & -0.30150000 & 1.07050000 & 0.76600000 \\ \mathrm{C} & -1.13950000 & 0.01890000 & 0.87720000 \\ \mathrm{C} & -2.47460000 & 0.13520000 & 0.97410000\end{array}$




\section{Supporting Information file}

\begin{tabular}{|c|c|c|c|}
\hline $\mathrm{C}$ & -3.13970000 & 1.31050000 & 0.94900000 \\
\hline C & -2.29640000 & 2.35680000 & 0.81300000 \\
\hline$C$ & -0.95970000 & 2.24600000 & 0.73080000 \\
\hline $\mathrm{N}$ & -4.42930000 & 1.41790000 & 1.03790000 \\
\hline $\mathrm{C}$ & -5.03400000 & 2.43300000 & 0.50180000 \\
\hline $\mathrm{C}$ & -5.11240000 & 0.51030000 & 1.66520000 \\
\hline $\mathrm{C}$ & -6.40780000 & 0.22710000 & 1.38540000 \\
\hline $\mathrm{C}$ & -7.12640000 & -0.70980000 & 2.02480000 \\
\hline $\mathrm{C}$ & -6.57350000 & -1.42990000 & 3.00770000 \\
\hline $\mathrm{C}$ & -5.29630000 & -1.18840000 & 3.32510000 \\
\hline $\mathrm{C}$ & -4.60110000 & -0.24790000 & 2.66540000 \\
\hline 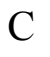 & 8.10870000 & -2.89280000 & -0.89410000 \\
\hline$C$ & 9.22220000 & -3.53720000 & -1.29940000 \\
\hline $\mathrm{C}$ & 9.26830000 & -4.81810000 & -1.70270000 \\
\hline 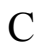 & 8.18430000 & -5.61380000 & -1.75400000 \\
\hline$C$ & 7.06080000 & -4.98870000 & -1.35630000 \\
\hline $\mathrm{C}$ & 7.03570000 & -3.70820000 & -0.94970000 \\
\hline $\mathrm{C}$ & 8.22040000 & -6.91070000 & -2.16340000 \\
\hline $\mathrm{C}$ & 7.18450000 & -7.41360000 & -2.89270000 \\
\hline $\mathrm{C}$ & 7.26580000 & -8.69410000 & -3.30420000 \\
\hline $\mathrm{C}$ & 8.29230000 & -9.48240000 & -2.97550000 \\
\hline $\mathrm{C}$ & 9.28250000 & -8.97470000 & -2.23750000 \\
\hline $\mathrm{C}$ & 9.29190000 & -7.68770000 & -1.83800000 \\
\hline$c$ & -6.21250000 & 2.92310000 & 0.95720000 \\
\hline$c$ & -6.84870000 & 3.97350000 & 0.41420000 \\
\hline $\mathrm{C}$ & -6.32770000 & 4.60370000 & -0.64500000 \\
\hline $\mathrm{C}$ & -5.16550000 & 4.15780000 & -1.1357000 \\
\hline $\mathrm{C}$ & -4.54980000 & 3.10760000 & -0.56910000 \\
\hline
\end{tabular}




\begin{tabular}{|c|c|c|c|}
\hline $\mathrm{C}$ & 13.95170000 & 1.94580000 & 0.94450000 \\
\hline $\mathrm{C}$ & 14.21440000 & 3.24510000 & 1.20430000 \\
\hline $\mathrm{C}$ & 13.25860000 & 4.02810000 & 0.70360000 \\
\hline $\mathrm{C}$ & 12.38080000 & 3.23480000 & 0.04710000 \\
\hline $\mathrm{C}$ & 15.26250000 & 3.73580000 & 1.87080000 \\
\hline $\mathrm{C}$ & 15.36130000 & 5.06840000 & 2.05060000 \\
\hline $\mathrm{C}$ & 14.41790000 & 5.92060000 & 1.56120000 \\
\hline $\mathrm{C}$ & 13.38920000 & 5.35240000 & 0.89080000 \\
\hline $\mathrm{O}$ & 14.63220000 & 1.00220000 & 1.28540000 \\
\hline $\mathrm{C}$ & 11.41700000 & 3.66710000 & -0.79680000 \\
\hline $\mathrm{C}$ & 12.74700000 & 1.95250000 & 0.30590000 \\
\hline $\mathrm{O}$ & 10.29310000 & -7.20760000 & -1.02010000 \\
\hline $\mathrm{C}$ & 11.07960000 & -8.10990000 & -0.27580000 \\
\hline $\mathrm{O}$ & 6.14690000 & -6.60290000 & -3.30290000 \\
\hline $\mathrm{C}$ & 5.39920000 & -6.95120000 & -4.44590000 \\
\hline $\mathrm{C}$ & 14.51740000 & 7.28570000 & 1.74740000 \\
\hline $\mathrm{O}$ & 15.47590000 & 7.67470000 & 2.39300000 \\
\hline $\mathrm{O}$ & 13.71250000 & 8.32980000 & 1.33500000 \\
\hline $\mathrm{C}$ & 12.56170000 & 8.26360000 & 0.54020000 \\
\hline $\mathrm{C}$ & 10.84920000 & 2.87270000 & -1.68910000 \\
\hline $\mathrm{N}$ & 10.42150000 & 2.20060000 & -2.53330000 \\
\hline $\mathrm{C}$ & 11.10970000 & 4.92980000 & -1.02190000 \\
\hline $\mathrm{N}$ & 10.84170000 & 6.03710000 & -1.23700000 \\
\hline $\mathrm{C}$ & 16.46020000 & 5.35410000 & 2.73120000 \\
\hline $\mathrm{N}$ & 17.44350000 & 5.51050000 & 3.32660000 \\
\hline $\mathrm{H}$ & 5.10190000 & -1.80120000 & -0.43390000 \\
\hline $\mathrm{H}$ & 11.12150000 & -1.59220000 & -0.60400000 \\
\hline $\mathrm{H}$ & 12.82200000 & -0.12460000 & 0.04700000 \\
\hline
\end{tabular}




\begin{tabular}{|c|c|c|c|}
\hline $\mathrm{H}$ & 3.18730000 & -1.43710000 & -0.18870000 \\
\hline 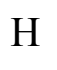 & 0.98900000 & -1.10050000 & 0.00000000 \\
\hline $\mathrm{H}$ & -0.77320000 & -1.01880000 & 0.92610000 \\
\hline $\mathrm{H}$ & -3.01650000 & -0.82660000 & 1.00630000 \\
\hline $\mathrm{H}$ & -2.66820000 & 3.39660000 & 0.82970000 \\
\hline $\mathrm{H}$ & -0.44100000 & 3.20490000 & 0.59110000 \\
\hline $\mathrm{H}$ & -6.94050000 & 0.70630000 & 0.54590000 \\
\hline $\mathrm{H}$ & -8.17140000 & -0.90730000 & 1.73010000 \\
\hline $\mathrm{H}$ & -7.15380000 & -2.20030000 & 3.54120000 \\
\hline $\mathrm{H}$ & -4.82590000 & -1.75360000 & 4.14800000 \\
\hline $\mathrm{H}$ & -3.58360000 & -0.08290000 & 3.06000000 \\
\hline $\mathrm{H}$ & 10.18830000 & -3.03370000 & -1.37670000 \\
\hline 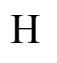 & 10.24550000 & -5.17030000 & -2.07300000 \\
\hline $\mathrm{H}$ & 6.10890000 & -5.53900000 & -1.27090000 \\
\hline $\mathrm{H}$ & 6.04660000 & -3.39210000 & -0.61150000 \\
\hline $\mathrm{H}$ & 6.48270000 & -9.15280000 & -3.92750000 \\
\hline $\mathrm{H}$ & 8.32170000 & -10.53400000 & -3.30770000 \\
\hline $\mathrm{H}$ & 10.09780000 & -9.66760000 & -1.97780000 \\
\hline $\mathrm{H}$ & -6.69390000 & 2.52890000 & 1.86880000 \\
\hline $\mathrm{H}$ & -7.79470000 & 4.33780000 & 0.85030000 \\
\hline$U$ & -6.84170000 & 5.46590000 & -1.10050000 \\
\hline $\mathrm{H}$ & -4.72850000 & 4.64700000 & -2.02310000 \\
\hline $\mathrm{H}$ & -3.63960000 & 2.78050000 & -1.10070000 \\
\hline $\mathrm{H}$ & 16.02310000 & 3.03920000 & 2.26500000 \\
\hline $\mathrm{H}$ & 12.59250000 & 5.96050000 & 0.47870000 \\
\hline $\mathrm{H}$ & 11.77890000 & -8.66370000 & -0.94050000 \\
\hline $\mathrm{H}$ & 11.69520000 & -7.51390000 & 0.43510000 \\
\hline $\mathrm{H}$ & 10.43060000 & -8.79480000 & 0.31430000 \\
\hline
\end{tabular}




$\begin{array}{lrrr}\mathrm{H} & 4.74330000 & -6.08710000 & -4.69630000 \\ \mathrm{H} & 6.07560000 & -7.13740000 & -5.30990000 \\ \mathrm{H} & 4.73940000 & -7.82150000 & -4.23470000 \\ \mathrm{H} & 12.22490000 & 9.31020000 & 0.36380000 \\ \mathrm{H} & 12.80350000 & 7.83890000 & -0.45890000 \\ \mathrm{H} & 11.73520000 & 7.75600000 & 1.08480000\end{array}$

\section{GH5}

$\begin{array}{lccl}\text { Symbol } & \mathbf{X} & \mathbf{Y} & \mathbf{Z} \\ \mathrm{C} & 9.24800000 & 1.19020000 & -0.44440000 \\ \mathrm{C} & 7.93590000 & 1.18830000 & -0.24730000 \\ \mathrm{C} & 7.56070000 & -0.08460000 & -0.39220000 \\ \mathrm{~N} & 8.54310000 & -0.87690000 & -0.65830000 \\ \mathrm{C} & 9.56190000 & -0.08660000 & -0.68310000 \\ \mathrm{~S} & 6.88190000 & 2.13930000 & 0.05720000 \\ \mathrm{C} & 5.74780000 & 1.19140000 & 0.05020000 \\ \mathrm{C} & 6.23000000 & -0.04540000 & -0.22150000 \\ \mathrm{C} & 10.89430000 & -0.06190000 & -0.83880000 \\ \mathrm{C} & 11.41790000 & 1.18210000 & -0.72690000 \\ \mathrm{~S} & 10.34290000 & 2.14730000 & -0.45940000 \\ \mathrm{C} & 4.44770000 & 1.52780000 & 0.27410000 \\ \mathrm{C} & 12.74790000 & 1.39560000 & -0.84200000 \\ \mathrm{C} & 3.48700000 & 0.58190000 & 0.25130000 \\ \mathrm{C} & 2.18540000 & 0.82810000 & 0.46280000 \\ \mathrm{C} & 1.68430000 & 2.05440000 & 0.71120000 \\ \mathrm{C} & 2.63840000 & 3.01300000 & 0.75070000 \\ \mathrm{C} & 3.95880000 & 2.76390000 & 0.54300000 \\ \mathrm{~N} & 2.40200000 & 4.22740000 & 1.02820000\end{array}$


Supporting Information file

\begin{tabular}{|c|c|c|c|}
\hline S & 3.78600000 & 5.21070000 & 1.01140000 \\
\hline $\mathrm{N}$ & 4.67060000 & 3.81080000 & 0.63570000 \\
\hline C & 0.34600000 & 2.23640000 & 0.88640000 \\
\hline $\mathrm{C}$ & -0.49200000 & 1.23540000 & 1.22720000 \\
\hline $\mathrm{C}$ & -1.81230000 & 1.39820000 & 1.41590000 \\
\hline $\mathrm{C}$ & -2.46320000 & 2.57230000 & 1.26680000 \\
\hline $\mathrm{C}$ & -1.62220000 & 3.56450000 & 0.90260000 \\
\hline $\mathrm{C}$ & -0.29890000 & 3.40910000 & 0.72690000 \\
\hline $\mathrm{N}$ & -3.73900000 & 2.72370000 & 1.44510000 \\
\hline $\mathrm{C}$ & -4.37540000 & 3.66130000 & 0.81300000 \\
\hline $\mathrm{C}$ & -4.37680000 & 1.93820000 & 2.25760000 \\
\hline $\mathrm{C}$ & -5.69460000 & 1.64820000 & 2.13180000 \\
\hline $\mathrm{C}$ & -6.36720000 & 0.83620000 & 2.96330000 \\
\hline $\mathrm{C}$ & -5.74110000 & 0.25870000 & 3.99520000 \\
\hline $\mathrm{C}$ & -4.43870000 & 0.51370000 & 4.16580000 \\
\hline $\mathrm{C}$ & -3.79110000 & 1.32670000 & 3.31570000 \\
\hline $\mathrm{C}$ & 8.51810000 & -2.15110000 & -0.85150000 \\
\hline $\mathrm{C}$ & 9.57320000 & -2.88030000 & -1.26940000 \\
\hline $\mathrm{C}$ & 9.56030000 & -4.20850000 & -1.47210000 \\
\hline $\mathrm{C}$ & 8.46890000 & -4.97250000 & -1.28210000 \\
\hline $\mathrm{C}$ & 7.40310000 & -4.26300000 & -0.86750000 \\
\hline $\mathrm{C}$ & 7.43660000 & -2.93510000 & -0.66480000 \\
\hline $\mathrm{C}$ & 8.44530000 & -6.31720000 & -1.48730000 \\
\hline $\mathrm{C}$ & 7.32910000 & -6.89700000 & -2.01250000 \\
\hline $\mathrm{C}$ & 7.35030000 & -8.22710000 & -2.22710000 \\
\hline $\mathrm{C}$ & 8.39890000 & -8.98390000 & -1.89460000 \\
\hline $\mathrm{C}$ & 9.47100000 & -8.39690000 & -1.35680000 \\
\hline $\mathrm{C}$ & 9.53840000 & -7.06490000 & -1.16470000 \\
\hline
\end{tabular}




\begin{tabular}{|c|c|c|c|}
\hline $\mathrm{C}$ & -5.50440000 & 4.24360000 & 1.28500000 \\
\hline $\mathrm{C}$ & -6.17200000 & 5.21610000 & 0.64350000 \\
\hline $\mathrm{C}$ & -5.73630000 & 5.66660000 & -0.53880000 \\
\hline $\mathrm{C}$ & -4.62640000 & 5.12270000 & -1.05120000 \\
\hline $\mathrm{C}$ & -3.97710000 & 4.15450000 & -0.38480000 \\
\hline $\mathrm{C}$ & 13.26360000 & 3.74110000 & -0.62570000 \\
\hline $\mathrm{C}$ & 14.43330000 & 4.40460000 & -0.65020000 \\
\hline $\mathrm{C}$ & 15.46050000 & 3.58820000 & -0.82920000 \\
\hline $\mathrm{C}$ & 14.98730000 & 2.32760000 & -0.92930000 \\
\hline $\mathrm{O}$ & 12.22720000 & 4.34700000 & -0.47760000 \\
\hline $\mathrm{C}$ & 15.75140000 & 1.22500000 & -1.11250000 \\
\hline $\mathrm{C}$ & 13.62080000 & 2.43070000 & -0.80170000 \\
\hline $\mathrm{C}$ & 14.75690000 & 5.69290000 & -0.53490000 \\
\hline S & 16.21460000 & 5.80090000 & -0.64670000 \\
\hline $\mathrm{C}$ & 16.54560000 & 4.37980000 & -0.83320000 \\
\hline $\mathrm{Cl}$ & 18.19180000 & 3.95330000 & -1.02140000 \\
\hline $\mathrm{O}$ & 10.63030000 & -6.49330000 & -0.54570000 \\
\hline $\mathrm{C}$ & 11.48960000 & -7.29320000 & 0.23420000 \\
\hline $\mathrm{O}$ & 6.26040000 & -6.13030000 & -2.42760000 \\
\hline $\mathrm{C}$ & 5.38400000 & -6.62920000 & -3.41240000 \\
\hline $\mathrm{C}$ & 17.06720000 & 1.18340000 & -1.22750000 \\
\hline $\mathrm{N}$ & 18.21300000 & 1.03710000 & -1.33950000 \\
\hline $\mathrm{C}$ & 15.33400000 & -0.02520000 & -1.20780000 \\
\hline $\mathrm{N}$ & 14.99160000 & -1.13050000 & -1.29280000 \\
\hline $\mathrm{H}$ & 5.58790000 & -0.92030000 & -0.31120000 \\
\hline $\mathrm{H}$ & 11.53980000 & -0.92860000 & -1.01350000 \\
\hline $\mathrm{H}$ & 13.23500000 & 0.43300000 & -1.01890000 \\
\hline $\mathrm{H}$ & 3.70780000 & -0.47550000 & 0.04190000 \\
\hline
\end{tabular}




\begin{tabular}{|c|c|c|c|}
\hline $\mathrm{H}$ & 1.53950000 & -0.05870000 & 0.36250000 \\
\hline 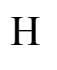 & -0.13570000 & 0.20820000 & 1.40370000 \\
\hline $\mathrm{H}$ & -2.36070000 & 0.46610000 & 1.63960000 \\
\hline $\mathrm{H}$ & -1.97850000 & 4.60370000 & 0.79090000 \\
\hline $\mathrm{H}$ & 0.21680000 & 4.32270000 & 0.39940000 \\
\hline $\mathrm{H}$ & -6.29110000 & 2.00860000 & 1.27600000 \\
\hline $\mathrm{H}$ & -7.43570000 & 0.62240000 & 2.78910000 \\
\hline $\mathrm{H}$ & -6.28300000 & -0.40810000 & 4.68550000 \\
\hline $\mathrm{H}$ & -3.90670000 & 0.06720000 & 5.02350000 \\
\hline $\mathrm{H}$ & -2.74180000 & 1.52400000 & 3.59530000 \\
\hline $\mathrm{H}$ & 10.52820000 & -2.42060000 & -1.53340000 \\
\hline $\mathrm{H}$ & 10.48590000 & -4.63970000 & -1.88870000 \\
\hline $\mathrm{H}$ & 6.46110000 & -4.76710000 & -0.59420000 \\
\hline $\mathrm{H}$ & 6.49580000 & -2.54370000 & -0.27140000 \\
\hline $\mathrm{H}$ & 6.49740000 & -8.75390000 & -2.68230000 \\
\hline $\mathrm{H}$ & 8.38000000 & -10.07440000 & -2.06110000 \\
\hline $\mathrm{H}$ & 10.30300000 & -9.06430000 & -1.08390000 \\
\hline $\mathrm{H}$ & -5.91050000 & 4.00310000 & 2.28270000 \\
\hline $\mathrm{H}$ & -7.07240000 & 5.66550000 & 1.09640000 \\
\hline $\mathrm{H}$ & -6.27680000 & 6.46310000 & -1.07570000 \\
\hline $\mathrm{H}$ & -4.26150000 & 5.46140000 & -2.03610000 \\
\hline $\mathrm{H}$ & -3.12020000 & 3.72810000 & -0.93440000 \\
\hline $\mathrm{H}$ & 14.07290000 & 6.53680000 & -0.38370000 \\
\hline 1 & 12.10260000 & -7.95900000 & -0.41260000 \\
\hline $\mathrm{H}$ & 12.18830000 & -6.61310000 & 0.77160000 \\
\hline $\mathrm{H}$ & 10.90720000 & -7.86410000 & 0.99150000 \\
\hline $\mathrm{H}$ & 4.71280000 & -5.79590000 & -3.72010000 \\
\hline H & 5.95570000 & -6.96490000 & -4.30640000 \\
\hline
\end{tabular}




\section{Supporting Information file}

$\mathrm{H}$ $4.74340000-7.43790000-2.99630000$

\section{Conflict of Interest}

No conflict of interest declared

\section{Acknowledgments}

The authors would like to acknowledge the support provided by King Fahd University of Petroleum and Minerals. This research article is a part of $\mathrm{PhD}$ thesis entitled "High-Throughput Screening to Enhance D- $\pi$-A Interaction in Functional Materials: Exploring the Synergistic Effects and Applications" of Mr. Muhammad Haroon s/o Ghulam Yaseen at KFUPM under the supervision of Prof. Dr. Muhammad Ramzan Saeed Ashraf Janjua. He can be reached at muhammad.yaseen@kfupm.edu.sa or phoolnagarr@gmail.com 